\title{
Research on the Minimum Size of Received Signal Strength Difference Localization Network
}

\author{
Fangli $\mathrm{Ma}^{1,2,3} \cdot$ Yang $\mathrm{Xu}^{3,4} \cdot$ Peng $\mathrm{Xu}^{3,4}(\mathbb{C}$
}

Received: 2 July 2021 / Accepted: 26 August 2021

(C) The Author(s) 2021

\begin{abstract}
The received signal strength difference (RSSD) localization is a kind of method to locate emission sources by measuring the differences of received signal strength level between the monitoring stations and is essentially the truth value ratios of measured signal strength. In the existing literatures, only the rule of RSSD localization circle of two monitoring stations and the geometric relation of RSSD localization circle of five monitoring stations were analyzed, but the number and the station layout of the minimum RSSD localization network have not been investigated. In the present work, first, based on the existing RSSD localization equation, the constants of the commonly used wave propagation models are provided. Then, the minimum RSSD localization network is proved through algebraic analysis, which is that four monitoring stations not distributed on a straight line can locate the signal source at one point. The relationship between the localization accuracy and the signal strength error of the RSSD location network with different scales is studied further and formulated as a nonlinear programming optimization problem. It is found that the localization stability of the network with four stations is poor, and there is a serious localization deviation outlier phenomenon. Therefore, the network with four stations is not available for radio monitoring networks with a signal strength error of \pm 5 to $\pm 10 \mathrm{~dB}$. The RSSD network with five stations is basically the minimum available size, and the RSSD network with nine stations can approach the localization accuracy of the angle of arrival (AOA) network with three stations.
\end{abstract}

Keywords Passive localization $\cdot$ RSSD $\cdot$ Minimum size of localization network $\cdot$ Localization accuracy $\cdot$ Signal strength error

\section{Introduction}

The passive radio localization methods based on the target transmitted signal, including the arrival angle localization method which takes the signal arrival angle as the localization parameter, the range relation localization method which takes the signal arrival distance as the localization

\section{Peng Xu}

pengxup@swjtu.edu.cn

1 School of Computing and Artificial Intelligence, Southwest Jiaotong University, Chengdu 610031, China

2 Sichuan Provincial Radio Monitoring Station, Chengdu 610031, China

3 National-Local Joint Engineering Laboratory of System Credibility Automatic Verification, Southwest Jiaotong University, Chengdu 610031, China

4 School of Mathematics, Southwest Jiaotong University, Chengdu 610031, China parameter, and the frequency difference localization method which takes the difference of the Doppler frequency shift between the observation point and the localization target as the localization parameter [1], are the methods to realize the location by measuring the parameters of the signal transmitted by the target. These methods belong to passive localization methods, because the localization equipment used does not transmit radio signals. According to different measurement parameters, the distance relation localization methods are divided into the time of arrival (TOA) method and the time difference of arrival (TDOA) method for measuring time, the received signal strength (RSS) [2-7] method and the received signal strength difference (RSSD) [8-11] method for measuring signal strength method. The RSS localization method, also known as power of arrival (POA), uses multiple radio receiving stations located in different locations to receive signals from a certain transmitting source at the same time, and calculates the location of the transmitting source by measuring the signal strength. 
The main drawback of this method is that the equivalent transmitting power of the signal source needs to be known in advance. Therefore, the RSS location is only applicable to the location of some signal sources with known transmission power and constant transmission power in use. It is mainly used for the location of early cellular network mobile stations and some indoor radio signal sources. The characteristics of these two localization scenarios are that the number of stations that can receive the same signal is small, and the distance between the monitoring station and the signal source is close, usually within several hundred meters. It is easy to establish a two-dimensional or three-dimensional Cartesian coordinate system.

The RSSD localization method, also known as the strength difference of arrival (SDOA) localization method, which does not need to know the equivalent transmitting power of the signal transmitting source in advance, nor does it need to measure the absolute signal strength received by each station, only needs to measure the difference of received signal strength between stations, has universality. The so-called radio signal strength difference here refers to the difference in the signal strength level expressed in decibels, essentially the ratio of the true value of the signal strength. In 2011, a two-dimensional RSSD localization model was proposed, which drew the distribution map of the localization circles with two monitoring stations under different level differences and the cross-plot of ten localization circles with the five-station location network, and presented the optimal nonlinear least squares solution of the RSSD localization problem [8]. In 2012, the maximum likelihood (ML) estimates for RSS and RSSD location under the assumption of lognormal path loss model were derived. It was proved that the ML estimation and the Crlach-Rao lower bound (CRLB) are the same [9], but the geometric relationship of the localization circle is not studied. In 2017, the strength difference localization algorithm of WLAN based on RSSD was studied, in which only considering the maximum number of access points as the three strongest signal points. Based on the hyperbolic intersection method, the iterative method was used to solve the localization coordinates [10]. The applicability of the three receiving stations and the hyperbolic intersection point method was worth further discussion. In 2018, using genetic algorithm to solve RSSD equations and calculate the position of signal source and the height of transmitting antenna was proposed [11]. There was no literature on the minimum size of RSSD location network.

Based on the background outlined above, the minimum size of RSSD location network is studied. First, based on the RSSD localization equation [8], the least stations proof is carried out by using algebraic analytic method. Then, the optimization method of nonlinear programming is used to simulate the practicability of several RSSD localization networks and compare them with AOA localization networks.

\section{RSSD Localization Equation}

Suppose that there are multiple radio monitoring stations in the RSSD localization network, and the location of the radio signal transmitting source is $\mathrm{O}$. It is easy to prove that the level difference of the radio signal strength received by the monitoring station $M_{i}$ and $M_{j}$ is expressed in (1) and (2).

$\Delta \mathrm{ST}_{i j}=\mathrm{ET}_{i}-\mathrm{PT}_{j}$,

$\Delta \mathrm{SM}_{i j}=\mathrm{EM}_{i}-\mathrm{EM}_{j}=\mathrm{PM}_{i}-\mathrm{PM}_{j}$,

where $\Delta \mathrm{ST}_{i j}$ and $\Delta \mathrm{SM}_{i j}$ are the theoretical predicted value and measured value of the signal strength level difference at $M_{j}$ and $M_{i}$ respectively, and the unit is dB.

$\mathrm{ET}_{i}, \mathrm{EM}_{i}, \mathrm{ET}_{j}$ and $\mathrm{EM}_{j}$ are the theoretical predicted values and measured values of the signal field strength of the two monitoring stations, respectively, and the units are all $\mathrm{dB} \mu \mathrm{V} / \mathrm{m}$.

$\mathrm{PT}_{i}, \mathrm{PM}_{i}, \mathrm{PT}_{j}$ and $\mathrm{PM}_{j}$ are, respectively, the theoretical predicted values and measured values of the signal received power of the two monitoring stations, and the unit is $\mathrm{dBm}$.

The theoretical prediction formula of signal received power is (3).

$\mathrm{PT}_{i, j}=\mathrm{EIRP}-\mathrm{Lp}_{i, j}(d)$,

where EIRP is omnidirectional transmitting power of the transmitting source, while $\mathrm{Lp}_{i}$ and $\mathrm{Lp}_{j}$ are propagation losses of signals from transmitting source $\mathrm{O}$ to monitoring stations $M_{i}$ and $M_{j}$, respectively.

The existing RSSD localization models [8-10] are based on the Plane Cartesian coordinate system. The distance formulas between transmitting source $\mathrm{O}$ and monitoring station $M_{i}$ and $M_{j}$ are (4) and (5).

$d_{i}=\sqrt{\left(x-x_{i}\right)^{2}+\left(y-y_{i}\right)^{2}}$,

$d_{j}=\sqrt{\left(x-x_{j}\right)^{2}+\left(y-y_{j}\right)^{2}}$,

where $\left(x_{i}, y_{i}\right)$ and $\left(x_{\mathrm{j}}, y_{\mathrm{j}}\right)$ are the rectangular coordinates of monitoring station $M_{i}$ and $M_{j}$ respectively, $(x, y)$ is the rectangular coordinates of transmitting source $\mathrm{O}, d_{i}$ and $d_{j}$ are the distances between transmitting source $\mathrm{O}$ and monitoring station $M_{i}$ and $M_{j}$, respectively, in unit of $\mathrm{km}$.

In Ref. [8], the general Eq. (6) of radio wave propagation was used to derive the localization equation.

$\operatorname{Pr}=\frac{A(\mathrm{ht}, \mathrm{hr}, f)}{d^{\gamma}} \mathrm{Pt}$.

Formula (6) is rewritten into formula (7) of radio wave propagation loss expressed in $\mathrm{dB}$. 
Table 1 Parameters setting of regular propagation model

\begin{tabular}{lll}
\hline Propagation model & $\gamma$ & $-10 \lg A(\mathrm{ht}, \mathrm{hr}, f) / \mathrm{dB}$ \\
\hline Free space & 2 & $32.45+20 \lg f$ \\
Ideal flat ground & 4 & $120-20 \lg (\mathrm{ht} \cdot \mathrm{hr})$ \\
Localized model [12] & $k_{4}+k_{5} \lg (\mathrm{ht})$ & $k_{1}+k_{2} \lg f+k_{3} \lg (\mathrm{ht})$ \\
Egli(hr $\leq 10 \mathrm{~m})$ & 4 & $78+20 \lg f-20 \lg (\mathrm{ht} \cdot \mathrm{hr})$ \\
Egli(hr $>10 \mathrm{~m})$ & 4 & $88+20 \lg f-20 \lg (\mathrm{ht} \cdot \mathrm{hr})$ \\
\hline
\end{tabular}

$\mathrm{Lp}=10 \gamma \lg d-10 \lg A(\mathrm{ht}, \mathrm{hr}, f)$.

In Eqs. (6) and (7), $A(\mathrm{ht}, \mathrm{hr}, f)$ is the constant determined by the transmitting antenna, the receiving antenna and the working frequency. ht and hr are the height of the transmitting antenna and the receiving antenna relative to the ground, respectively, in meters (m), and $\gamma$ is the path loss index of the radio wave propagation.

According to Ref. [12], constants $A(\mathrm{ht}, \mathrm{hr}, f)$ and path loss index $\gamma$ of common wave propagation models can be listed, as shown in Table 1.

$$
\begin{aligned}
\mathrm{PT}_{i j}= & \operatorname{EIRP}-10 \gamma \lg \left[\left(x-x_{i, j}\right)^{2}+\left(y-y_{i, j}\right)^{2}\right] \\
& +10 \lg A\left(\mathrm{ht}, \mathrm{hr}_{i, j}, f\right) .
\end{aligned}
$$

Based on Eqs. (1)-(8), it is assumed $\Delta \mathrm{SM}_{i j}=\Delta \mathrm{ST}_{i j}+\delta_{i j}$, $\delta_{i j}$ is the error between the measured value and the theoretical predicted value, and the localization Eq. (9) can be obtained.

$10 \gamma \lg \frac{\left(x-x_{j}\right)^{2}+\left(y-y_{j}\right)^{2}}{\left(x-x_{i}\right)^{2}+\left(y-y_{i}\right)^{2}}+10 \lg \frac{A\left(\mathrm{ht}, \mathrm{hr}_{i}, f\right)}{A\left(\mathrm{ht}, \mathrm{hr}_{j}, f\right)}=\Delta \mathrm{SM}_{i j}-\delta_{i j}$.

In Eq. (9), the measured value of signal strength level difference $\Delta \mathrm{SM}_{i j}$ is the observed quantity, and the rectangular coordinate $(x, y)$ of the transmitting source $\mathrm{O}$ is the unknown quantity.

Assumed that

$\Omega_{i j}=10^{\frac{\Delta \mathrm{SM}_{i j}-\delta_{i j}}{10 \gamma}-\frac{1}{\gamma} \lg \frac{A(\mathrm{hth}, \mathrm{hr}, \mathrm{f} f)}{A\left(\mathrm{hth}, \mathrm{r}_{i} f\right)} .}$

Then, Eq. (9) is simplified to Eq. (11).

$\frac{\left(x-x_{j}\right)^{2}+\left(y-y_{j}\right)^{2}}{\left(x-x_{i}\right)^{2}+\left(y-y_{i}\right)^{2}}=\Omega_{i j}$.
If $\Omega_{i j} \neq 1$, the standard Eq. (12) of the circle can be obtained.

$$
\left(x-\frac{x_{j}-\Omega_{i j} x_{i}}{1-\Omega_{i j}}\right)^{2}+\left(y-\frac{y_{j}-\Omega_{i j} y_{i}}{1-\Omega_{i j}}\right)^{2}=\left(\frac{\sqrt{\Omega_{i j}} d_{i j}}{1-\Omega_{i j}}\right)^{2} .
$$

Equation (12) represents a circle with $\left(\frac{x_{j}-\Omega_{i j} x_{i}}{1-\Omega_{i j}}, \frac{y_{j}-\Omega_{i j} y_{i}}{1-\Omega_{i j}}\right)$ as the center and $\frac{\sqrt{\Omega_{i j}} d_{i j}}{1-\Omega_{i j}}$ as the radius.

When $\Omega_{i j}=1$, the line represented by Eq. (13) can be obtained, which is the perpendicular line of the connection between two points $M_{i}$ and $M_{j}$.

$y=\frac{x_{j}}{y_{i}} x+\frac{x_{i}^{2}+y_{i}^{2}-x_{j}^{2}-y_{j}^{2}}{2 y_{i}}$.

If there are $n$ monitoring stations that can receive a certain signal and the signal strength level difference between the waves reaching these stations can be measured, then the localization equations composed of $n(n-1) / 2$ equations can be obtained at (14).

$$
\left\{\begin{array}{rl}
\frac{\left(x-x_{2}\right)^{2}+\left(y-y_{2}\right)^{2}}{\left(x-x_{1}\right)^{2}+\left(y-y_{1}\right)^{2}} & =\Omega_{12} \\
\frac{\left(x-x_{3}\right)^{2}+\left(y-y_{3}\right)^{2}}{\left(x-x_{1}\right)^{2}+\left(y-y_{1}\right)^{2}} & =\Omega_{13} \\
\cdots \cdots & \cdots \\
\frac{\left(x-x_{n}\right)^{2}+\left(y-y_{n}\right)^{2}}{\left(x-x_{n-1}\right)^{2}+\left(y-y_{n-1}\right)^{2}} & =\Omega_{(n-1) n}
\end{array} .\right.
$$

\section{Analytical Proof of the Least Stations}

The minimum size of RSSD localization network refers to the number and layout of stations in the RSSD localization network that can locate the signal source when there is only one signal source at a certain frequency in the monitoring coverage area. Algebraic analytic proof of RSSD location will be performed by starting with three sites and gradually increasing the number of sites until the minimum number of sites in the RSSD location network for a single point of location is found. The coordinates of the signal source is set as $\left(x_{0}, y_{0}\right)$.

\subsection{Localization of Three Monitoring Stations}

$$
\left\{\begin{array}{c}
x=\frac{2 x_{0}^{2} x_{2} y_{3}^{2}+x_{0} x_{2}^{2} x_{3}^{2}-x_{0} x_{2}^{2} y_{3}^{2}-2 x_{0} x_{2} x_{3}^{3}-2 x_{0} x_{2} x_{3} y_{3}^{2}+x_{0} x_{3}^{4}+2 x_{0} x_{3}^{2} y_{3}^{2}+x_{0} y_{3}^{4}}{4 x_{0}^{2} y_{3}^{2}-4 x_{0} x_{2} y_{3}^{2}+x_{2}^{2} x_{3}^{2}+x_{2}^{2} y_{3}^{2}-2 x_{2} x_{3}^{3}-2 x_{2} x_{3} y_{3}^{2}+4 x_{2} x_{3} y_{3} y_{0}+x_{3}^{4}+2 x_{3}^{2} y_{3}^{2}-4 x_{3}^{2} y_{3} y_{0}+y_{3}^{4}-4 y_{3}^{3} y_{0}+4 y_{3}^{3} y_{0}^{2}} \\
+\frac{2 x_{2}^{2} x_{3} y_{3} y_{0}-2 x_{2} x_{3}^{2} y_{3} y_{0}-2 x_{2} y_{3}^{3} y_{0}+2 x_{2} y_{3}^{2} y_{0}^{2}}{4 x_{0}^{2} y_{3}^{2}-4 x_{0} x_{2} y_{3}^{2}+x_{2}^{2} x_{3}^{2}+x_{2}^{2} y_{3}^{2}-2 x_{2} x_{3}^{3}-2 x_{2} x_{3} y_{3}^{2}+4 x_{2} x_{3} y_{3} y_{0}+x_{3}^{4}+2 x_{3}^{2} y_{3}^{2}-4 x_{3}^{2} y_{3} y_{0}+y_{3}^{4}-4 y_{3}^{3} y_{0}+4 y_{3}^{3} y_{0}^{2}} \\
y=\frac{2 x_{0}^{2} x_{3}^{2} y_{3}-2 x_{0}^{2} x_{2} x_{3} y_{3}+2 x_{0}^{2} y_{3}^{3}+2 x_{0} x_{2}^{2} x_{3} y_{3}-2 x_{0} x_{2} x_{3}^{2} y_{3}-2 x_{0} x_{2} y_{3}^{3}-x_{2}^{2} x_{3}^{2} y_{0}+x_{2}^{2} y_{3}^{2} y_{0}+2 x_{2} x_{3}^{3} y_{0}+2 x_{2} x_{3} y_{3}^{2} y_{0}}{4 x_{0}^{2} y_{3}^{2}-4 x_{0} x_{2} y_{3}^{2}+x_{2}^{2} x_{3}^{2}+x_{2}^{2} y_{3}^{2}-2 x_{2} x_{3}^{3}-2 x_{2} x_{3} y_{3}^{2}+4 x_{2} x_{3} y_{3} y_{0}+x_{3}^{4}+2 x_{3}^{2} y_{3}^{2}-4 x_{3}^{2} y_{3} y_{0}+y_{3}^{4}-4 y_{3}^{3} y_{0}+4 y_{3}^{2} y_{0}^{2}} \\
-\frac{2 x_{2} y_{3} y_{0}^{2}+x_{3}^{4} y_{0}+2 x_{3}^{2} y_{3}^{2} y_{0}-2 x_{3}^{2} y_{3} y_{0}^{2}+y_{3}^{4} y_{0}-2 y_{3}^{3} y_{0}^{2}}{4 x_{0}^{2} y_{3}^{2}-4 x_{0} x_{2} y_{3}^{2}+x_{2}^{2} x_{3}^{2}+x_{2}^{2} y_{3}^{2}-2 x_{2} x_{3}^{3}-2 x_{2} x_{3} y_{3}^{2}+4 x_{2} x_{3} y_{3} y_{0}+x_{3}^{4}+2 x_{3}^{2} y_{3}^{2}-4 x_{3}^{2} y_{3} y_{0}+y_{3}^{4}-4 y_{3}^{3} y_{0}+4 y_{3}^{2} y_{0}^{2}}
\end{array}\right.
$$


When the number of monitoring stations is 3 , there are two groups of solutions to (14). When the known numbers are concrete numbers, it is easy to solve. However, when the known numbers are algebraic, the expressions are complicated.

To save space, only the minimum number of stations required for RSSD localization is demonstrated:

(1) As shown in Fig. 1, for the RSSD localization network composed of arbitrary triangle vertices $S_{1}, S_{2}$ and $S_{3}$, the coordinates of the three monitoring stations are set as $S_{1}(0,0), S_{2}\left(x_{2}, 0\right)$ and $S_{3}\left(x_{3}, y_{3}\right)$, respectively, then (15) is obtained. There are two groups of solutions for (15), the first group $s$ is $\left(x_{0}, y_{0}\right)$, the second group is (16).

$$
\left\{\begin{array}{l}
\frac{\left(x-x_{2}\right)^{2}+y^{2}}{x^{2}+y^{2}}=\frac{\left(x_{0}-x_{2}\right)^{2}+y_{0}^{2}}{x_{0}^{2}+y_{0}^{2}} \\
\frac{\left(x-x_{3}\right)^{2}+\left(y-y_{3}\right)^{2}}{x^{2}+y^{2}}=\frac{\left(x_{0}-x_{3}\right)^{2}+\left(y_{0}-y_{3}\right)^{2}}{x_{0}^{2}+y_{0}^{2}}
\end{array} .\right.
$$

(2) As shown in Fig. 1, when the three monitoring stations $S_{1}, S_{2}$ and $S_{4}$ are on a straight line, the coordinates of the three monitoring stations are set as $S_{1}(0,0), S_{2}\left(x_{2}\right.$, $0)$ and $S_{4}\left(x_{4}, 0\right)$, respectively, and (17) is obtained.

$$
\left\{\begin{array}{l}
\frac{\left(x-x_{2}\right)^{2}+y^{2}}{x^{2}+y^{2}}=\frac{\left(x_{0}-x_{2}\right)^{2}+y_{0}^{2}}{x_{0}^{2}+y_{0}^{2}} \\
\frac{\left(x-x_{4}\right)^{2}+y^{2}}{x^{2}+y^{2}}=\frac{\left(x_{0}-x_{4}\right)^{2}+y_{0}^{2}}{x_{0}^{2}+y_{0}^{2}}
\end{array} .\right.
$$

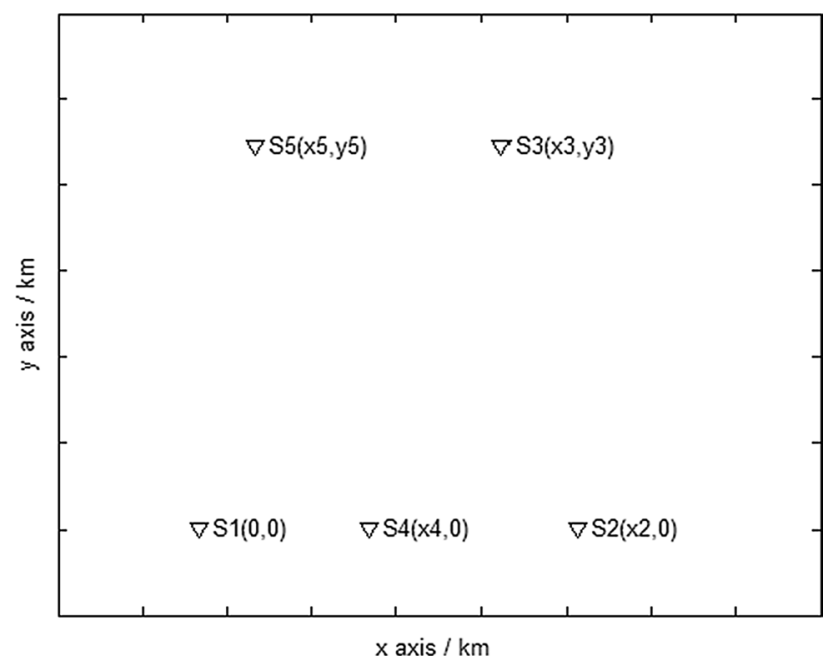

Fig. 1 Distribution of RSSD location network
The solutions of (17) are, respectively, $\left(x_{0}, y_{0}\right)$ and $\left(x_{0}\right.$, $\left.-y_{0}\right)$. It is easy to prove that there are two points symmetrical to the line for the localization curve of the RSSD localization network with no less than three monitoring stations distributed in a straight line.

Here, $\left(x_{0},-y_{0}\right)$ and (16) are the mirror points of the signal source $\left(x_{0}, y_{0}\right)$. That is, if a radio source with the same power and antenna height at $\left(x_{0}, y_{0}\right)$ and the mirror points, each of the three monitoring stations will receive the same radio signal strength, respectively.

Thus, it can be seen that any three monitoring stations which receive the same signal can determine two localization points, one of which is the point of the signal source, and the other is the false point.

\subsection{Localization of Four Monitoring Stations}

As shown in Fig. 1, when four stations $S_{1}(0,0), S_{2}\left(x_{2}\right.$, $0), S_{3}\left(x_{3}, y_{3}\right)$ and $S_{5}\left(x_{5}, y_{5}\right)$ are not on the same straight line, there must be four three-station localization subnets with different mirror points of their solutions. Therefore, the point $\left(x_{0}, y_{0}\right)$ where the signal source is located can be located.

Then, the localization network composed of four radio monitoring stations which are not on the same straight line can realize RSSD localization, is proved.

There are three possibilities for the arrangement of four stations RSSD localization network. First, convex four stations network, is a localization network with four stations located at the vertexes of a convex quadrilateral. Second, concave four stations network, is namely four stations located at a concave quadrilateral vertex RSSD localization network. At last, enhanced-edge four stations network, that is, one station is added to one side of the triangle-vertice stations. The schematic diagram of their positioning is shown in Fig. 2.

Therefore, it is proved by algebraic analytic method that four stations which are not on a straight line can locate the signal source. However, there are two sets of solutions for the localization equations based on the data of three stations, one of which is the coordinate of the location where the signal source is located, and the other is the false point. However, due to the difference between the radio wave propagation model and the reality, as well as the existence of the measurement error of the radio signal strength, the localization deviation exists. The localization circles of four stations that are not on the same straight line often intersect not at one point, but at multiple points near the location of the signal source, as shown in Fig. 3. This results in no solution to the localization system, because the common intersection point of all the localization curves does not exist. Therefore, the algebraic analytic method is not practical. 


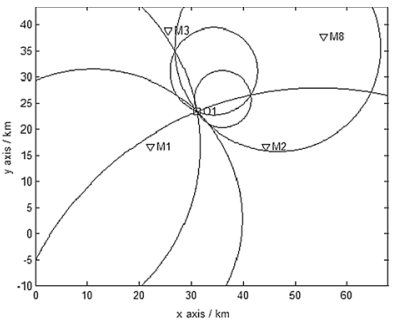

(a) Convex 4 stations network (inside)

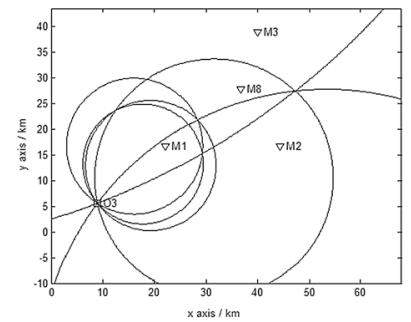

(c) Concave 4 stations network

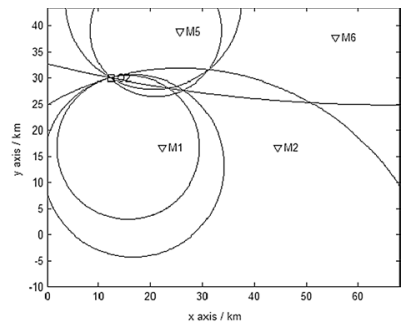

(b) Convex 4 stations network (outside)

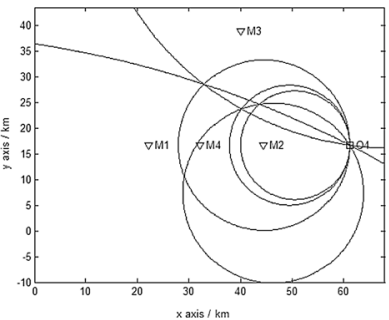

(d) Enhanced-edge 4 stations network
Fig. 2 Array of four stations not in a straight line

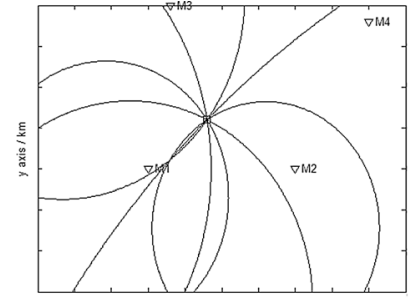

$\mathrm{xaxis} / \mathrm{km}$

(a) the ideal unbiased case

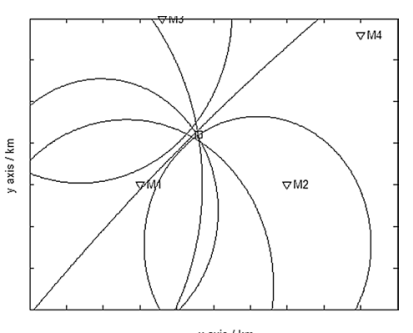

(b) the actual biased case
Fig. 3 RSSD localization schematic diagram

\section{Optimization Method of RSSD Localization}

When there are prediction errors of wave propagation and measurement errors of signal strength, the solution may not be obtained for algebraic analytic method, as shown in Fig. 3b. The drawing method can judge the intersection points manually, but it is difficult to solve them automatically. Here, an optimal localization modeling method based on unconstrained nonlinear programming is adopted $[13,14]$.

Taking the cumulative quantity of the difference between the predicted value and the measured value of the signal strength level difference from the hypothetical location to any two stations as the objective function, also taking the longitude and latitude of the location of the transmitting source as the decision variable. The optimization objective function of RSSD is (18). $f(x, y)=\operatorname{Min} \sum_{i=1 \ldots K: j=1 \ldots K}^{i>j}\left|\delta_{i j}\right|^{N}$.

By substituting Eq. (9) into (18), the optimal RSSD localization model (19) can be obtained.

$$
f(x, y)=\operatorname{Min} \sum_{i=1 \ldots K ; j=1 \ldots K}^{i>j}\left|\begin{array}{l}
\Delta \mathrm{SM}_{i j}-10 \gamma \lg \frac{\left(x-x_{j}\right)^{2}+\left(y-y_{j}\right)^{2}}{\left(x-x_{i}\right)^{2}+\left(y-y_{i}\right)^{2}} \\
-10 \lg \frac{A\left(\mathrm{ht}, \mathrm{hr}_{i}, f\right)}{A\left(\mathrm{ht}, \mathrm{hr}_{j}, f\right)}
\end{array}\right|^{N}
$$

In (18) and (19), $N$ is a positive integer. When $N=2$, the above optimal RSSD location based on unconstrained nonlinear programming becomes the least square RSSD location.

The issues of the RSSD localization are similar to that of the TDOA localization [14], also are the nonlinear programming problems. There are many methods to solve them, whereas the non-classical methods are not appropriate because of its convergence, which is difficult to be used for comparing the performances of different localization networks.

\section{Relationship Between Strength Errors and Localization Errors}

Considering that the level measurement accuracy of the radio monitoring receiver is required to be $\pm 1.5 \mathrm{~dB}$ (firstlevel and second-level) and $\pm 3 \mathrm{~dB}$ (third-level) [15], and the accuracy of the radio monitoring station is also related to the antenna and the feeder, the level measurement accuracy of the monitoring station is actually lower than that of the monitoring receiver. According to the data of acceptance testing in standard site conducted by radio management organization for many years, the measurement errors of VHF/UHF fixed radio monitoring system in standard site can reach $\pm 3 \mathrm{~dB}$. According to the verification testing of the fixed radio monitoring stations in use in recent years, the measurement errors of the actual fixed radio monitoring stations can reach $\pm 5 \mathrm{~dB}$ at the best and $\pm 10 \mathrm{~dB}$ at the worst.

The two-dimensional coordinates of the signal source and the height of the antenna are set first to predict the difference of the radio signal strength received between the two monitoring stations. Then, the optimal localization model (19) of RSSD is solved by point-by-point two-dimensional search method [13], and the localization results are obtained. The curve of the variation of localization accuracy with the signal strength error is plotted to compare the performance of RSSD localization networks with different array forms. In which, the signal source coordinates are sets and the location of the signal source 
is selected by Monte Carlo method, that is, the coordinate of the signal source is a random variable evenly distributed in a certain area.

To obtain the minimum available size of RSSD localization network, it is assumed that the measurement error of the signal strength of the monitoring station is $\pm \Delta \mathrm{dB}$, that is the measurement error is evenly distributed in the range of $-\Delta-\Delta \mathrm{dB}$. The location of the signal sources selected by Monte Carlo method, and the simulation times are 2000 times. The localization performances of the location network of four stations (three layouts), five stations, eight stations and nine stations are simulated, respectively.

For convenience of calculation, RSSD localization network is set as a north-south phalanx or the triangle array with one side in the longitudinal or latitudinal direction, and the limits of geographical coordinates in the four directions of each station are localization network boundaries. Within the boundaries is the network area, referred to as inside area of the network, and $50 \%$ of the distance outside the boundary line is extrapolated as the adjacent area of the off-network. Signal strength error range localization error.

For three RSSD localization network with four stations which can realize single point localization in theory, the measurement error of signal strength is set as $0 \mathrm{~dB}, \pm 0$. $5 \mathrm{~dB}, \pm 1 \mathrm{~dB}, \ldots, \pm 5 \mathrm{~dB}$, a total of 11 cases. The simulation results are shown in Fig. 3. In Fig. 4, when the signal strength measurement error is 0 to $\pm 5 \mathrm{~dB}$, the root mean square of the localization errors of the three four-station networks are as follows:

(1) The in-network localization error of the convex four stations network is very large, which is $30-80 \%$ of the side length of the localization network; the error of the

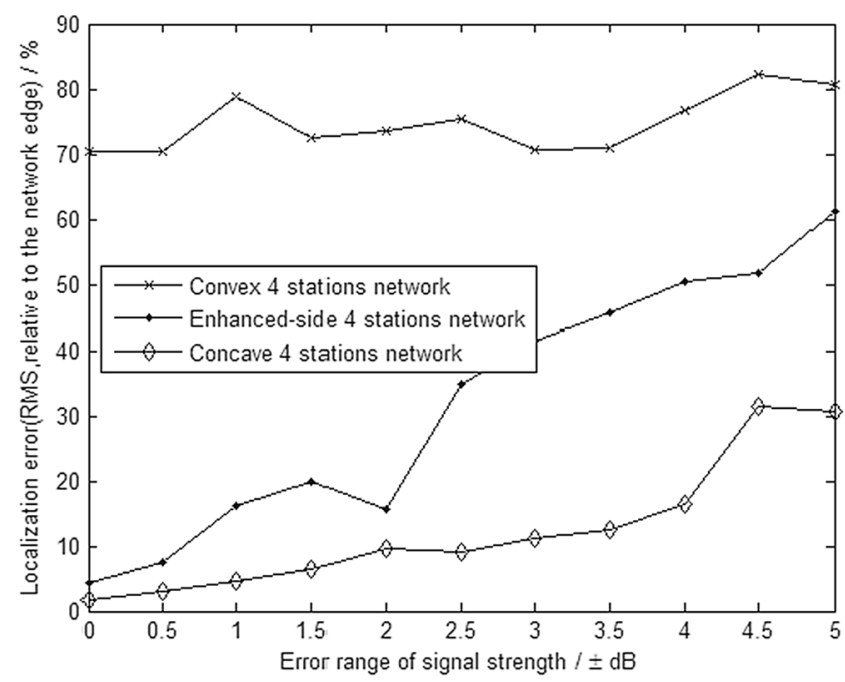

(a) The inside area of the network near region outside the network is a little smaller, in $9-40 \%$.

(2) The in-network localization error of the concave four stations network is within $32 \%$, and its error of the near area outside the network can reach $50 \%$. The localization error of the enhanced-edge four stations network is basically between the convex four stations network and the concave four stations network.

For the RSSD localization networks with 5 stations, 8 stations and 9 stations, the relationship curves between strength measurement errors and localization errors are drawn through simulation, and the measurement errors of signal strength are set as $0 \mathrm{~dB}, \pm 1 \mathrm{~dB}, \pm 2 \mathrm{~dB}, \ldots, \pm 10 \mathrm{~dB}$, a total of 11 cases. The simulation results are shown in Fig. 5. As can be seen from Fig. 5, when the actual measurement errors of signal strength in the range of \pm 5 to $\pm 10 \mathrm{~dB}$ for the fixed radio monitoring station, the root mean square of the localization errors of the three localization networks are as follows:

(1) The in-network localization errors of the five-station phalanx network (including a central station) and the eight-station phalanx network (excluding central station) are close to each other, about $12-35 \%$ of the network side length. The same index of the nine-station network (including a central station) is 6-13\%

(2) The location error of the near region outside the network of the five-station phalanx network (including a central site) is about $27-54 \%$ of the network side length. The same index of eight-station phalanx network (including a central station) and nine-station phalanx network is close, about $12-28 \%$, and that of

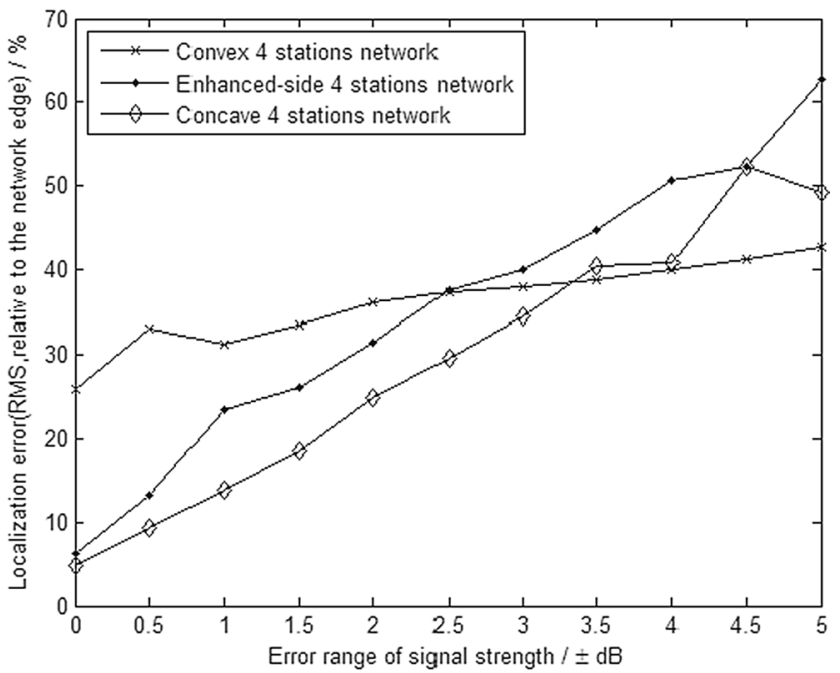

(b) The adjacent off-network area

Fig. 4 Comparison of accuracy of three types RSSD localization networks with four stations 


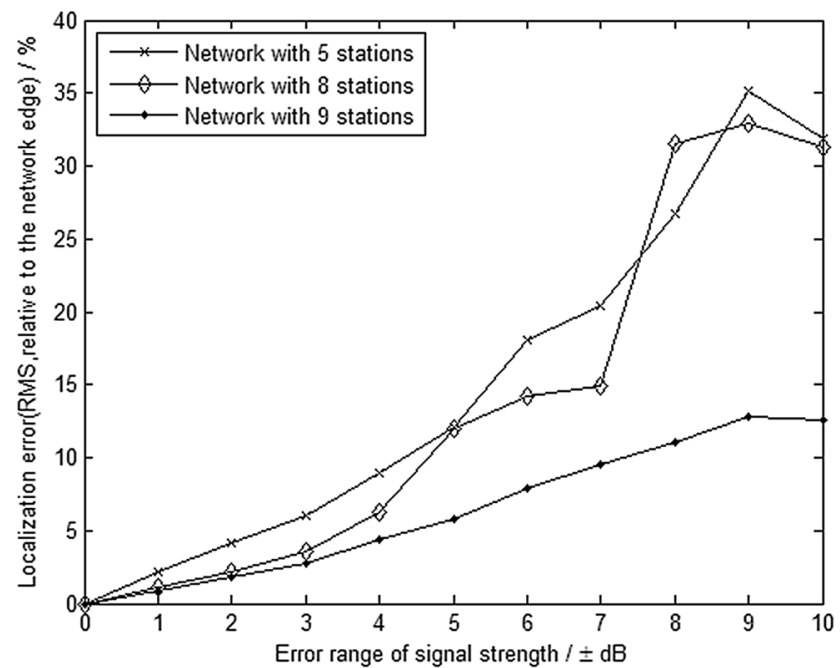

(a) The inside area of the network

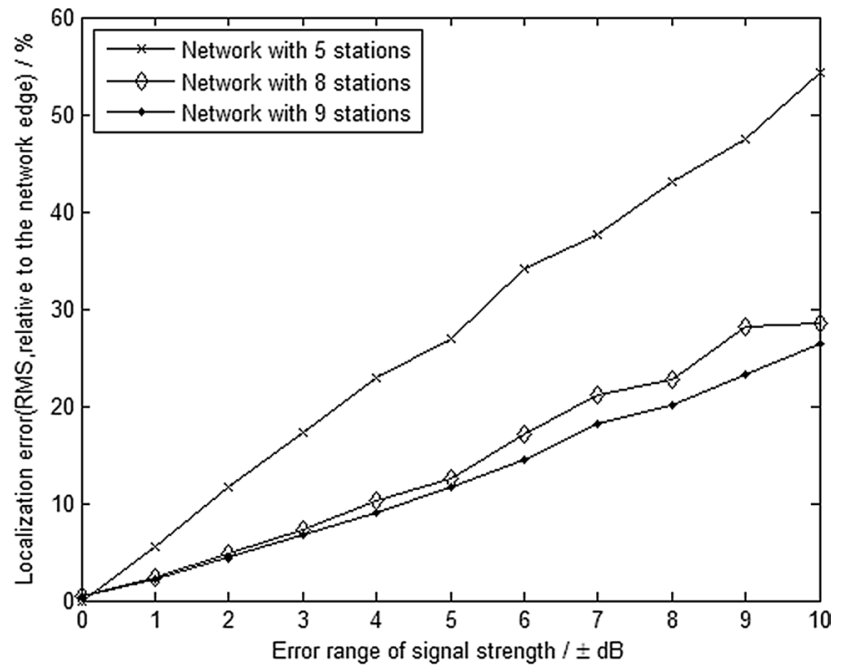

(b) The adjacent off-network area

Fig. 5 Comparison of accuracy of RSSD localization networks with 5-9 stations

the nine-station network is slightly less than that of the eight-station network.

First of all, the localization error is mainly decided by strength error in the RSSD algorithm, which can be proved by principles. In addition, it is apparent that under the same station layout, the localization accuracy increases with the improvement of the strength measurement accuracy in the simulations. In the same type of station layout (with the central point and without central point), the more stations, the higher the localization accuracy. Under the same requirements of inside-network localization accuracy, the network with a central station requires fewer stations than the network without a central station. For example, the insidenetwork localization accuracy of 5-station square network (including the central station) and 8-station square network (excluding the central station) is close.

\section{Accuracy Discussing About the Localization Network with Four Stations}

The localization accuracy of the three types of four-station RSSD localization networks shown in Fig. 4 is also poor when the signal strength error is small, especially the localization accuracy of the convex four-station network is poor.

Further numerical results show that when the signal strength deviation exceeds a certain limit, the localization deviation may produce outlier phenomenon, that is the anchor point may become near the point where another solution determined by one of the three stations in the network is located, as shown in Fig. 6. In combination with
Fig. 2, it is easy to see that the outliers in Fig. 6 all have another intersection point determined by the corresponding three stations. The outlier phenomenon of localization deviation is not found in AOA and TDOA localization, but also unique to RSSD localization. It is determined by the characteristic of RSSD localization that the localization curves of three stations intersect at two points.

The poor localization accuracy of the convex four stations network is from that the localization outlier phenomenon is very serious. The simulation with rectangular four stations network, in which $\pm 1 \mathrm{~dB}$ signal strength measurement error range, and a total of nine source positions within the network and outside but near the network were set up, including $\mathrm{A}, \mathrm{B}$, and $\mathrm{C}$ which located on the straight line where stations $\mathrm{M}_{1}$ and $\mathrm{M}_{2}$ are located, $\mathrm{A}, \mathrm{D}$, and $G$ which located on the inner side of the line where stations $\mathrm{M}_{1}$ and $\mathrm{M}_{5}$ are located, $\mathrm{C}, \mathrm{F}$ and I which located on the outside of the line where the stations $\mathrm{M}_{2}$ and $\mathrm{M}_{6}$ are located. Each of the 15 simulations, with different symbols to represent the location of the localization results. Too many simulations will result in serious overlap of drawing symbols. The localization results are shown in Fig. 7. As can be seen from Fig. 7, the location errors located in the center of the network and near the monitoring stations are small, and that of the other regions are very large. As can be seen from the localization results at $\mathrm{D}$ and $\mathrm{F}$, although all being close to the side of the network, the error in the network is obviously greater than that in the outside near region.

It is easy to understand the simulation localization results as shown in Fig. 4. The localization accuracy of the convex four stations network is very poor, and the inner region of the network is obviously worse than the outside near region. 


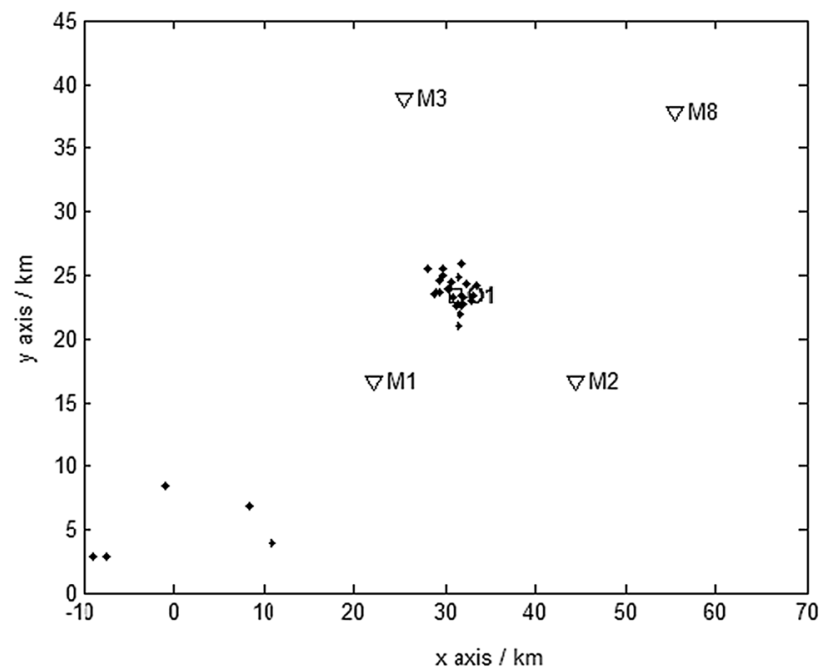

(a) Convex 4 stations network (inside)

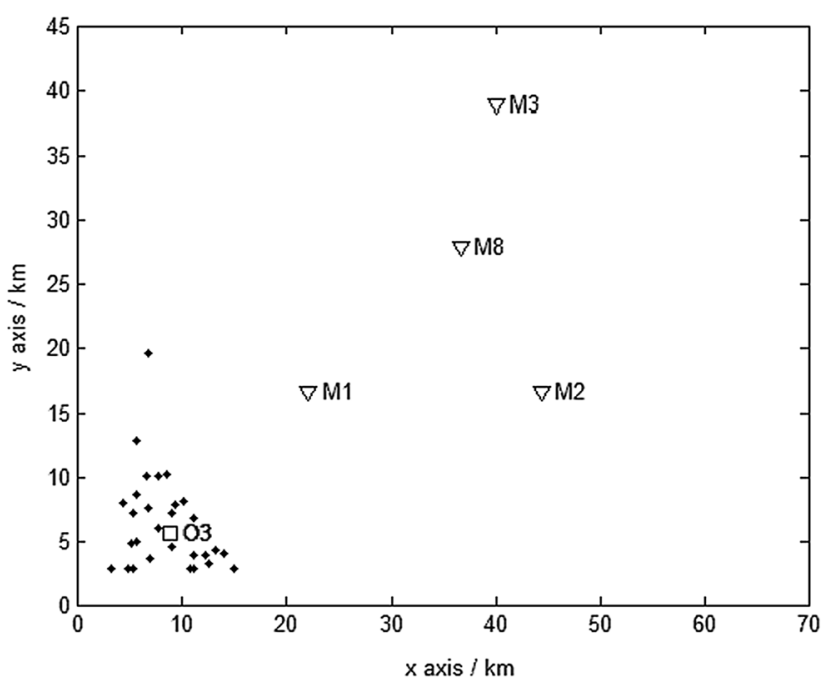

(c) Concave 4 stations network

Fig. 6 Error outlier phenomenon of RSSD localization network

\section{Comparison with AOA Localization}

TDOA has high localization accuracy, but it is not suitable for narrowband signals. RSSD localization is the same as AOA localization, which is commonly used in broadband signals and narrowband signals. To compare with AOA localization, AOA localization network is deployed at the same site as RSSD localization network for simulation, and the results are as follows:

(1) In Fig. 8a, the curve of accuracy variation with direction-finding error range of AOA localization network of three stations (the same sites as the three peripheral

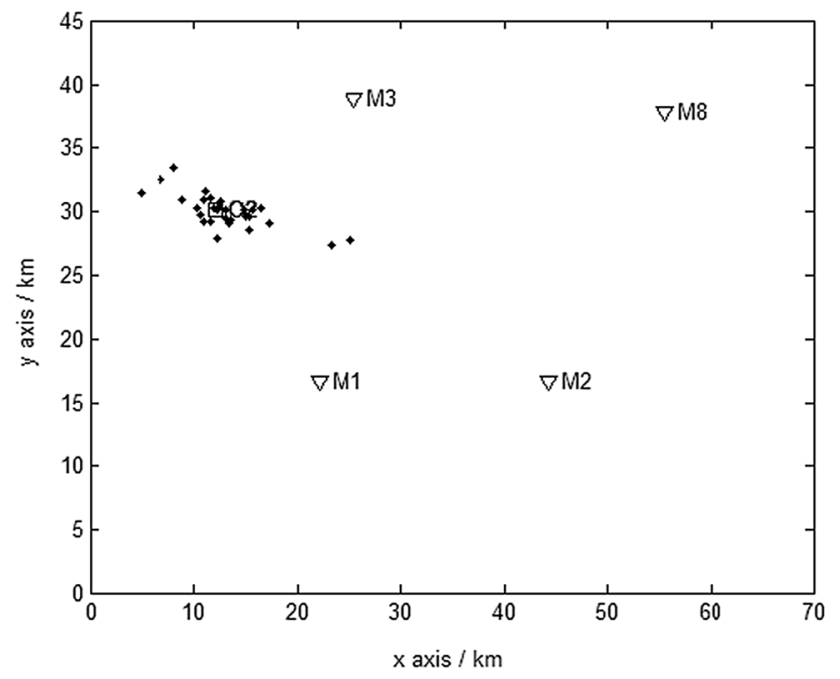

(b) Convex 4 stations network (outside)

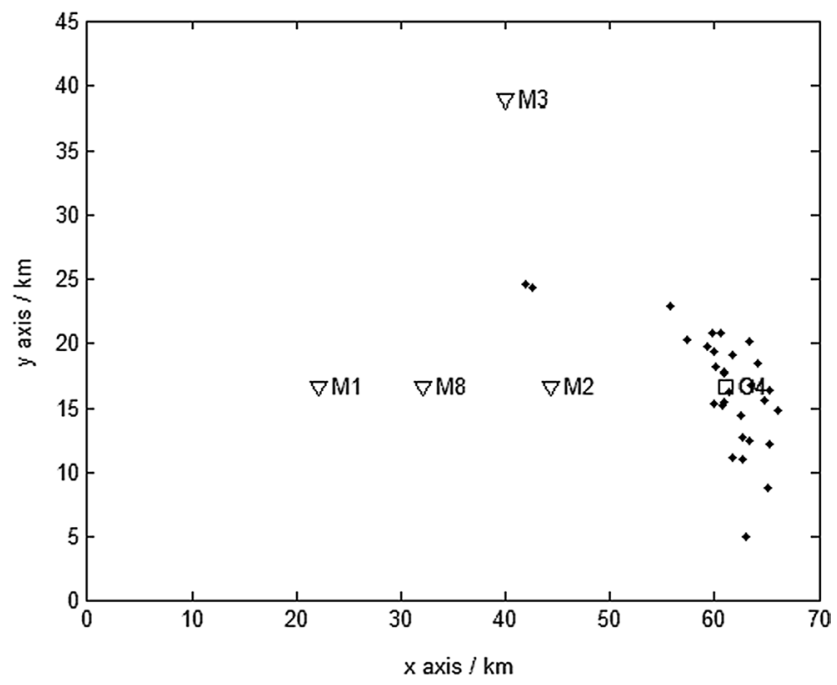

(d) Enhanced-edge 4 stations network

stations of RSSD localization network of concave four stations) was given.

(2) In Fig. 8b, the curve of accuracy variation with direction-finding error range of AOA localization network of four stations (the same sites as the RSSD localization network of convex four stations) was also given.

By comparing Fig. 8 with Fig. 4, only the RSSD location network with nine stations can approach the localization accuracy of AOA location network with three stations.

First of all, the site selection of AOA localization network is a complicated project, and the station cost with AOA function is high. The advantages of RSSD localization network, 


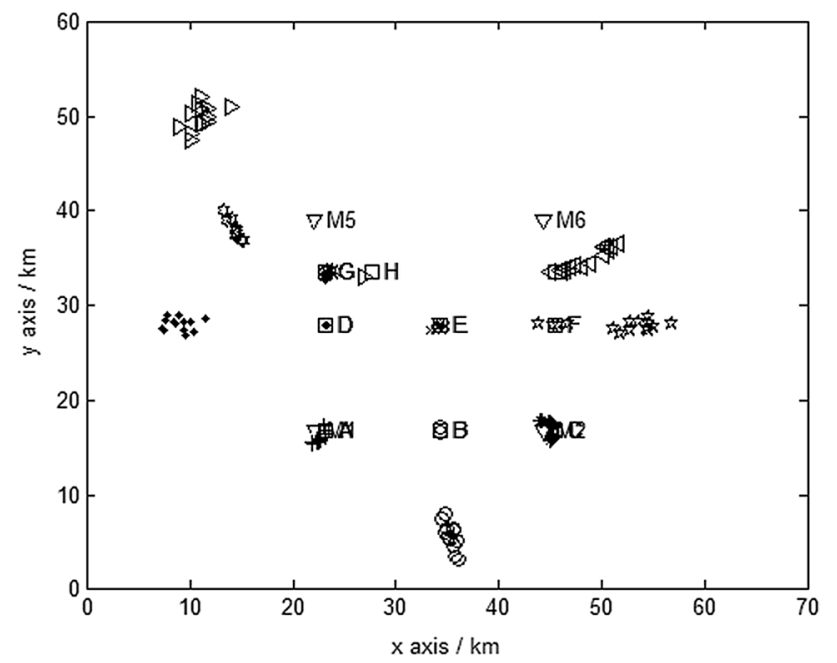

Fig. 7 RSSD localization network

on one hand, are that the whole cost will be less, although the number of stations is more than that of AOA localization network. On the other hand, for deployed AOA stations (direction-finding stations), AOA/RSSD hybrid localization can be used to further improve localization accuracy.

\section{Conclusions}

To further explore the location mechanism of RSSD, based on the existing RSSD location equation, the location network composed of four stations which are not on the

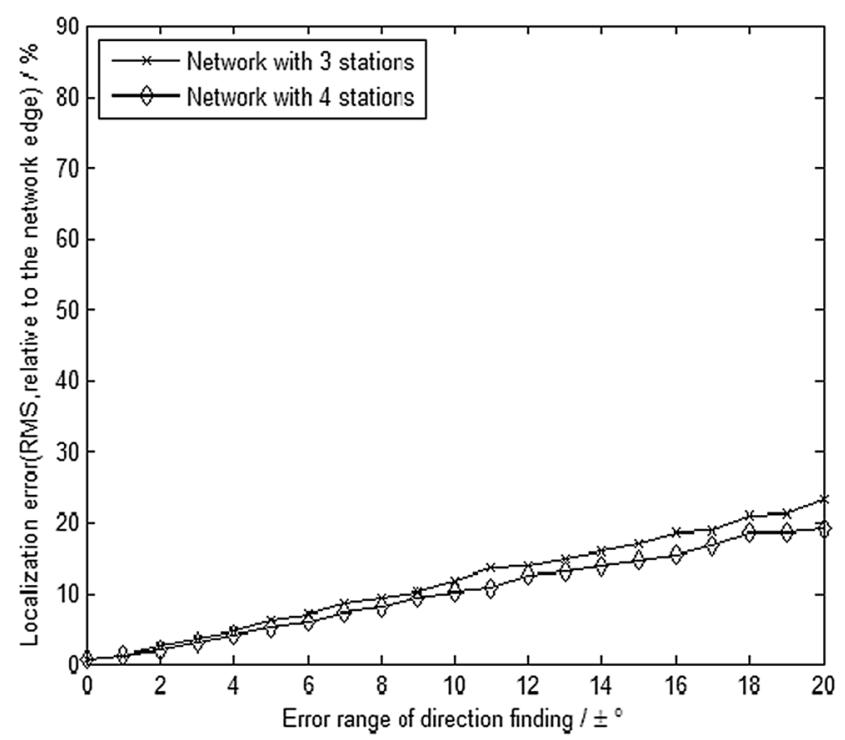

(a) The inside area of the network same line can realize the single point location, was proved through the algebraic analysis method. The optimized RSSD model was then proposed, in which the localization accuracy of RSSD localization networks with four stations, five stations, eight stations, and nine stations, were compared with the variation of the signal strength level error ranges, respectively. At the same time, the proposed model was also compared with the AOA location network. The following conclusions have been drawn:

(1) In an ideal situation, the RSSD location network composed of four monitoring stations which are not on a straight line can realize single point localization. However, due to the signal strength error, the localization error is very large, so it is not applicable.

(2) For the fixed radio monitoring network which is actually set up and the measurement error range of the field strength may reach \pm 5 to $\pm 10 \mathrm{~dB}$, the localization error inside the network of five stations square network (including the central site), are close to that of eight stations square network (excluding center site), about $0-35 \%$ of the network side. And the localization error of nine stations square network (including the central site) is $0-13 \%$. The localization error in the near area outside the network of the five stations square network (including the central site) is about $0-54 \%$ of the network side length, and the proximity localization error of the eight stations square network (including the central site) and the nine stations square network is about $0-28 \%$, in which the nine stations square network is slightly less than the eight stations square network.

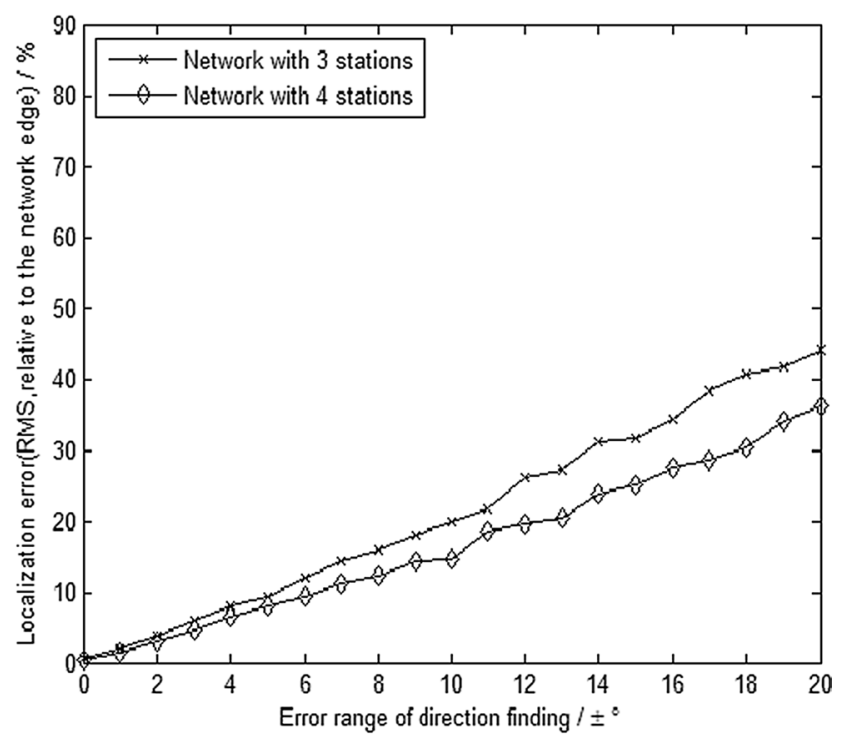

(b) The adjacent off-network area

Fig. 8 Accuracy of AOA localization network 
(3) Five-station localization network is the minimum available RSSD location network, and nine-station RSSD location network can approach the localization accuracy of three stations AOA location network.

The AOA localization method requires high setting environment for the direction-finding station, otherwise its direction-finding error will be large. The TDOA localization method is not suitable for narrowband signals. These problems can be avoided for the RSSD localization method. RSSD localization has not been widely used in radio monitoring networks because of its unsatisfactory performance for fixed radio monitoring.

On one hand, RSSD localization technology is sensitive to the change of signal strength. On the other hand, the fixed monitoring antenna is often affected by the tower and limited by the polarization mode, and it is difficult for the fixed monitoring station to accurately measure the radio signal strength. With the development of technology, the grid monitoring technology brings the possibility of densely distributed stations in the radio monitoring network [16], and optimization monitoring and direction-finding technology also brings the possibility of accurate measurement of signal strength by radio monitoring stations [17].

Acknowledgements This work is supported by National Science Foundation of China (Grant No. 61976130), Humanities and social sciences research project of the Ministry of Education of P. R. China (Grant No. 20XJCZH016), Sichuan Key Technology Research and Development Program (Grant No. 2020YJ0270), the Fundamental Research Funds for the Central Universities (Grant No. 2682021GF012). The authors thank the National Radio Monitoring Center of China and the Western Branch of China Academy of Information and Communications Technology for providing the statistics of in-service radio monitoring station test verification.

\section{Declarations}

Conflict of interest The authors declare that they do not have any commercial or associative interest that represents a conflict of interest in connection with the work submitted.

Open Access This article is licensed under a Creative Commons Attribution 4.0 International License, which permits use, sharing, adaptation, distribution and reproduction in any medium or format, as long as you give appropriate credit to the original author(s) and the source, provide a link to the Creative Commons licence, and indicate if changes were made. The images or other third party material in this article are included in the article's Creative Commons licence, unless indicated otherwise in a credit line to the material. If material is not included in the article's Creative Commons licence and your intended use is not permitted by statutory regulation or exceeds the permitted use, you will need to obtain permission directly from the copyright holder. To view a copy of this licence, visit http://creativecommons.org/licenses/by/4.0/.

\section{References}

1. Tian, Z.C., Liu, C.F.: Passive locating technology. National Defense Industrial Press, Beijing (2014)

2. Wang, K.D.: Geo-location in urban areas using signal strength repeatability. IEEE Commun. Lett. 5(10), 411-413 (2001)

3. Fan, P.Z., Deng, P., Liu, L.: Cellular wireless location. Electronic Industrial Press, Beijing (2002)

4. Sun, L., Zhang, S., Li, Z., et al.: Wireless sensor networks: theory and applications. Tsinghua University Press, Beijing (2018)

5. Dag, T., Arsan, T.: Received signal strength based least squares lateration algorithm for indoor localization. Comput. Electr. Eng. 66, 114-126 (2018)

6. Alam, N., Balaie, A.T., Dempster, A.G.: Dynamic path loss exponent and distance estimation in a vehicular network using Doppler effect and received signal strength. In: Proc. of VTC2010-Fall. Ottawa, ON, Canada: IEEE, pp. 1-5 (2010)

7. Uluskan, S., Filik, T.: A geometrical closed form solution for RSS based far-field localization: direction of exponent uncertainty. Wirel. Netw. 25(1), 215-227 (2019)

8. Wang, S.C., Inkol, R.: A near-optimal least squares solution to received signal strength difference based geolocation. In: Proc. ICASSP 2011. Prague, Czech Republic: IEEE, pp. 2600-2603 (2011)

9. Wang, S.C., Inkol, R., Jackson, B.R.: Relationship between the maximum likelihood emitter location estimators based on received signal strength (RSS) and received signal strength difference (RSSD). In: Proc. 2012 26th Biennial Symposium on Communications (QBSC). Kingston, On, Canada: IEEE, pp. 64-69 (2012)

10. Jing, H.F., Wang, B.: Design and implementation of location service based on signal strength difference. In: Proc. 13th International Conference on Intelligent Environments. Seoul, South Korea: IEEE, pp. 1-5 (2017)

11. Su, L.C., Wang, J.B.: Radio source locating method based on signal-strength-difference in gridded radio monitoring system. J. Chengdu Univ. Inf. Technol. 33(4), 391-394 (2018)

12. Xie, Y.X.: Radio wave propagation - principles and applications. In: Edition 1. Posts \& Telecom Press of China, p. 139 (2008)

13. Ma, F.L., Xu, Y., Xu, P.: 2D-TDOA passive location based on geodetic longitude and latitude. J. Commun. 40(05), 136-143 (2019)

14. Ma, F.L., Xu, Y., Xu, P.: A nonlinear programming based universal optimization model of TDOA passive location. In: Proc. of 12th International Conference on Intelligent Systems and Knowledge Engineering (ISKE2017). Nanjing: IEEE press, pp. 399-402 (2017)

15. GB/T32401-2015: Technical requirements and test methods for VHF/UHF frequency radio monitoring receivers. Standardization Administration of China (2015)

16. Zhang, J.S., Lv, M.F., Li, D.: An ICA based method for grid radio signal monitoring. Acta Sci. Nat. Univ. Pekin. 12, 307-314 (2017)

17. Qiu, C.Y., Bai, Y.J.: A method of radio monitoring and direction finding. In: Chinese patent application number: 201610462421.5, date of application: 2016.06.23 (2016)

Publisher's Note Springer Nature remains neutral with regard to jurisdictional claims in published maps and institutional affiliations. 